\title{
Expansile dentigerous cyst invading the entire maxillary sinus: a case report
}

\author{
Ju-Yeon Cho, Ki-Young Nam \\ Department of Dentistry, Dongsan Medical Center, Keimyung University, Daegu, Korea
}

\begin{abstract}
J Korean Assoc Oral Maxillofac Surg 2012;38:245-8)
Reported cases of a large dentigerous cyst involving the whole maxillary sinus are uncommon. A 22-year-old female patient suffering from swelling of the right infraorbital area and cheek with dull pain was referred to our department. Findings on computed tomography (CT) and magnetic resonance imaging (MRI) revealed a huge mass containing a displaced maxillary third molar involving the right maxillary sinus as a whole, with partial erosion of the posterior sinus cortical bone. Under general anesthesia, the mass was enucleated using the Caldwell-Luc approach, and, following histopathological analysis, was diagnosed as a dentigerous cyst. The case was followed for a period of seven years, and no evidence of sinus infection or recurring cyst formation was observed during that time.
\end{abstract}

Key words: Dentigerous cyst, Maxillary sinus, Ectopic third molar

[paper submitted 2012. 2. 9 / revised 2012. 2. 10 / accepted 2012. 5. 3]

\section{Introduction}

Originating with the separation of the follicle from around the crown of an unerupted tooth, the dentigerous cyst develops from fluid accumulation between the reduced enamel epithelium and the enamel surface ${ }^{1}$. Cystic development is usually related to unerupted third molar or maxillary canine, and it has the tendency of displacing the related tooth. A cyst formed around the maxillary third molar may invade the maxillary sinus, growing unnoticed to such extensive size as to occupy a considerable portion of the maxillary sinus. As the cyst grows, the bony walls overlying the cyst thin out, giving rise to an egg shell sensation upon palpation and possibly transmitting pressure to the walls of the sinus; thus causing ophthalmologic and nasal symptoms to develop ${ }^{2}$. Some untreated dentigerous cysts may - in rare cases - grow

\section{Ki-Young Nam}

Department of Dentistry, Dongsan Medical Center, Keimyung University,

56, Dalseong-ro, Jung-gu, Daegu 700-712, Korea

TEL: +82-53-250-7807 FAX: +82-53-250-7802

E-mail:nkyp@dsmc.or.kr

@) This is an open-access article distributed under the terms of the Creative Commons Attribution Non-Commercial License (http:/lcreativecommons.org/licenses/by-nc/3.0/), which permits unrestricted non-commercial use, distribution, and reproduction in any medium, provided the original work is properly cited. large and have the potential to develop into an odontogenic tumor such as ameloblastoma or to become malignant as in oral squamous cell carcinoma and mucoepidermoid carcinoma.

The standard treatment for a dentigerous cyst involves surgical enucleation and extraction of the cyst-associated impacted or unerupted tooth ${ }^{3,4}$, but long time for followup is required to rule out postoperative recurrence and postoperative cystic development ${ }^{5}$.

The present case of an extensive dentigerous cyst involving the whole maxillary sinus in a 22-year-old female is not common; the lesion was enucleated by the Caldwell-Luc approach supported by computed tomography (CT) and magnetic resonance imaging (MRI) scan. The case was followed for a period of 7 years, and there was no evidence of sinus infection or recurring cyst formation. Thus, we present the case with literature reviews.

\section{Case report}

A 22-year-old female patient under orthodontic treatment at a local clinic was referred to our department for further evaluation. The patient suffered from swelling and dull pain around the right infraorbital area, with partial right-sided nasal obstruction and watering of the right eye. The intraoral 
examination revealed fluctuant mass palpating on the buccal vestibule from the first premolar to the second molar; though the mobility of the teeth could not be measured owing to fixed orthodontic appliances, teeth from the canine to the second molar showed negative response in the percussion test and turned out to be vital in electric pulp test. On Water's view, the right maxillary third molar was dislocated into the right maxillary sinus with the haziness of sinus. Thinned alveolar cortical bone around the lateral wall of the sinus was also observed.(Fig. 1. A) The CT scan showed an enlarged cystic lesion measuring $5.2 \mathrm{~cm}$ horizontally, $4.3 \mathrm{~cm}$ vertically, and $4 \mathrm{~cm}$ sagittally; a partially disrupted posterior sinus wall with some obliteration of the retro-maxillary flat plane caused by expansive mass was also noted.(Fig. 1. B) MRI revealed an expansile mass invading into the anterior masticatory space, but the dark rim of the posterior to the mass implicated the preservation of the posterior wall of the sinus. There was no evidence of orbital extension.(Fig. 2)
There were no abnormal findings in the patient's general examination, so enucleation of cyst of the right maxilla was performed using the Caldwell-Luc approach under general anesthesia. After periosteal reflection, the cystic sac was identified, and cystic mass containing yellowish fluid was detected from the thinned buccal cortical bone. The cyst was easily dissected together with the ectopic third molar from the sinus walls except the posterior wall-which was partially eroded - to the posterior wall of the sinus. The enucleated mass measured $5.3 \times 4.2 \mathrm{~cm}$ and had an oval shape. The crown of the third molar was located within the lumen of the cyst, and its root remained outside.(Fig. 3. A) The excised mass was submitted for histopathological analysis, revealing a relatively uniform layer of non-keratinized, stratified, squamous epithelial lining cell.(Fig. 3. B) A diagnosis of the dentigerous cyst was made, and the postoperative status of the patient was uneventful. The patient remained symptomfree over a postoperative follow-up period of 7 years.
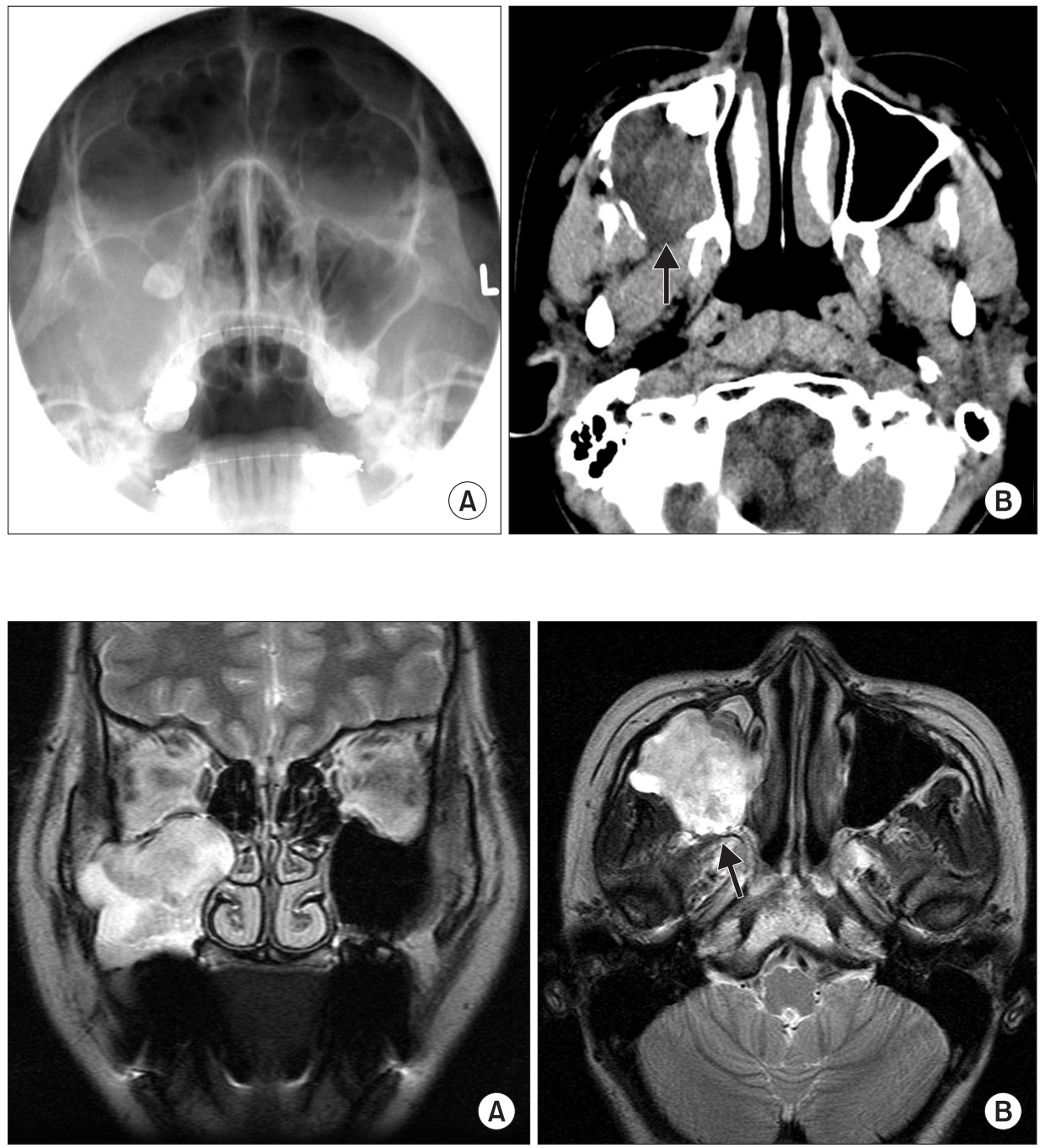

Fig. 2. A. Pre-operative frontal magnetic resonance (MR) image. B. Hypointense dark rim (arrow) is seen at posterior border of the mass in axial MR image.

Ju-Yeon Cho et al: Expansile dentigerous cyst invading the entire maxillary sinus: a case report. $J$ Korean Assoc Oral Maxillofac Surg 2012 

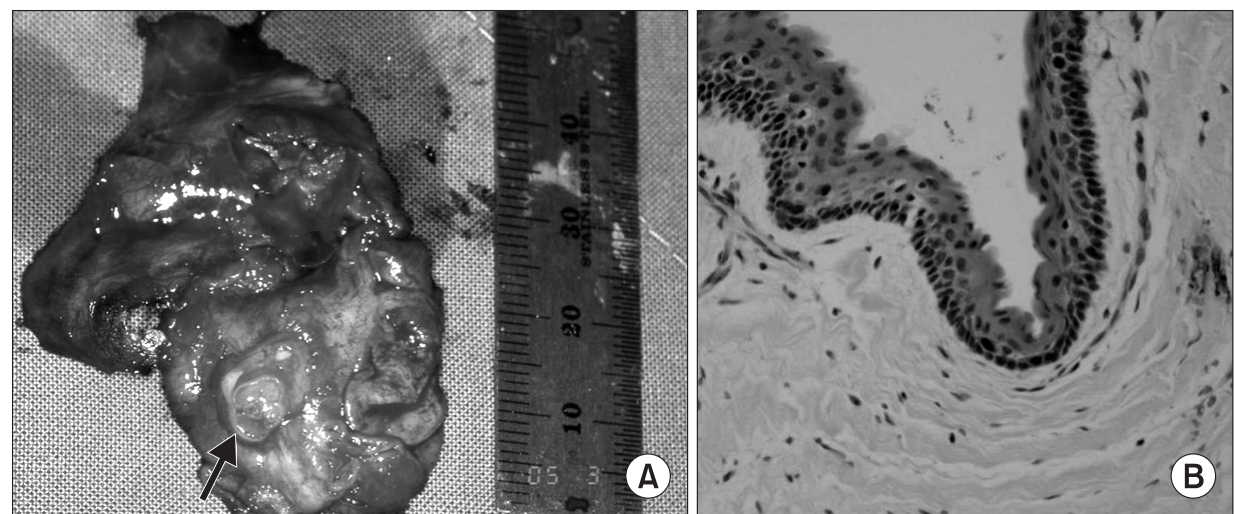

Fig. 3. A. The excised mass with a displaced third molar contained (arrow). B. Histopathologically, nonkeratinizing squamous epithelial lining, cholesterol clefts and Rushton body are seen (H\&E staining, $\times 200$ ).

$J u$-Yeon Cho et al: Expansile dentigerous cyst invading the entire maxillary sinus: a case report. $J$ Korean Assoc Oral Maxillofac Surg 2012

\section{Discussion}

As the most common type of developmental odontogenic cyst, dentigerous cyst is defined as an odontogenic cyst derived from reduced enamel epithelium surrounding the crown of an unerupted tooth ${ }^{1}$. It makes up about $20 \%$ of epithelium-lined cysts in the jaw, and it is commonly associated with unerupted mandibular or maxillary third molars or maxillary cuspids. It occurs at any age but has peak incidence in patients from the 10-30 year-old age group ${ }^{6,7}$. When the maxillary sinus is invaded, symptoms usually occur late in the process; the cyst usually remains asymptomatic but may produce painless bony expansion or facial asymmetry if it becomes large ${ }^{2,8}$. If inflamed, however, it becomes symptomatic, exhibiting classic signs of sinus disease including swelling, facial pain, headache, and nasolacrimal obstruction $^{2,3,8,9}$. A large maxillary cyst involved the whole sinus in the present case. Since it can transmit pressure to the walls of the sinus, ophthalmologic and nasal symptoms may develop. Epiphora could occur owing to the pressure to the nasolacrimal canal from the cyst associated with an ectopic canine in the maxillary sinus ${ }^{2}$, and orbital proptosis could develop from a dentigerous cyst in the maxillary sinus associated with a displaced tooth ${ }^{7}$. As a differential diagnosis, ameloblastoma is a benign epithelial odontogenic tumor consisting of cells similar to the enamel organ of dental follicle. It has similar clinical radiographic characteristics to the dentigerous cyst such as relatively rapid growth, painless facial swelling, and predilection at the mandibular molar area. Differential diagnosis between the two diseases is important in view of the clinical, radiological, prognostic, and therapeutic similarities. Note, however, that the age group in the incidence of ameloblastoma is younger compared to that of the cyst and odontogenic epithelium of dentigerous cyst, and dental follicle around an impacted tooth is usually replaced by squamous epithelium ${ }^{10}$; hence the low potential for transit to ameloblastoma. In addition, root resorption adjacent to the lesion is a critical factor in the radiographic differential diagnosis between the tumor and cysts in the jaw; that of ameloblastoma is higher than dentigerous cyst ${ }^{5,10}$.

Dentigerous cysts usually occur as well-circumscribed, unilocular radiolucencies surrounding the crown of a tooth and corticated border. In the present case, the entire borders of the lesion could not be clearly defined by plain radiograph such as Water's and panoramic views owing to image overlapping related to the hard palate and petrous bone of the skull $^{10,11}$. The border of cysts may be rendered ambiguous by secondary infection or expansion to the posterior sinus wall; hence the need for 3-dimensional images. The CT scan showed cystic expansion to masticatory space, bony erosion of pterygoid wall, and thinned outer cortex. Since the unclear posterior border of cyst was detected, however, MR imaging was done to confirm the posterior border of the mass and to distinguish between malignant tumor and inflammation. In MR imaging, no changes in signal intensity at the masticatory space were detected; the dark rim of the hypointense signal located in the posterior mass reflected the preserved posterior wall of the maxillary sinus ${ }^{12,13}$.(Fig. 2. B)

Despite the disadvantages damaging to the vitality of engaged tooth and tissues, cyst enucleation is the most common procedure to prevent the development of benign or malignant tumors caused by cystic remnants. The Caldwell-Luc approach was reported to be the most common treatment when dentigerous cysts are associated with an impacted tooth within the maxillary sinus ${ }^{14,15}$. Marsupialization is another advisable treatment to preserve the cyst-associated tooth and promote its eruption ${ }^{16}$; note, however, that the major disadvantage of marsupialization is the recurrence or persistence of the lesion ${ }^{17}$. We performed total enucleation of the cyst and displaced tooth using the Caldwell-Luc operation followed 


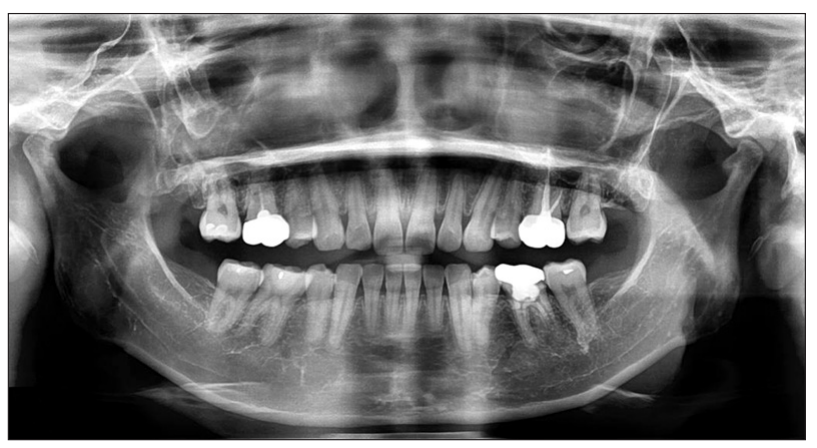

Fig. 4. Post-operative panoramic view after 7 years. $J u$-Yeon Cho et al: Expansile dentigerous cyst invading the entire maxillary sinus: a case report. J Korean Assoc Oral Maxillofac Surg 2012

by drainage to the nasoantral window rather than intraoral marsupialization because the posterolateral walls of the maxillary sinus were found to be eroded in part. No specific complications have been noted for 7 years after surgery (Fig. 4), but further follow-up is still needed to exclude the possibility of recurrence or postoperative maxillary cyst.

\section{References}

1. Kim SG, Park CY, Kang TH, Jang HS. Clinicopathologic study on cysts and postoperative cyst in maxillary sinus. J Korean Assoc Maxillofac Plast Reconstr Surg 2000;22:568-76.

2. Altas E, Karasen RM, Yilmaz AB, Aktan B, Kocer I, Erman Z. A case of a large dentigerous cyst containing a canine tooth in the maxillary antrum leading to epiphora. J Laryngol Otol 1997;111:641-3.
3. Motamedi MH, Talesh KT. Management of extensive dentigerous cysts. Br Dent J 2005;198:203-6.

4. Kim KW, Lee JH. Clinical study of cysts in the jaws. J Korean Assoc Maxillofac Plast Reconstr Surg 1999;21:166-73.

5. Park TW. Clinico-radiological study of cyst of the jaw. Korean J Oral Maxillofac Radiol 1983;13:163-8.

6. Hong SP, Lee JI, Shin HI, Choi HR, Kim EC, Park HR. Contemporary oral and maxillofacial pathology. Seoul: Jeong Won Publishing Co.; 1999.

7. Im CY. Color atlas of oral pathology. Seoul: Korea Medical Publishing Co.; 1992.

8. Albright CR, Hennig GH. Large dentigerous cyst of the maxilla near the maxillary sinus: report of case. J Am Dent Assoc 1971;83:1112-5.

9. Avitia S, Hamilton JS, Osborne RF. Dentigerous cyst presenting as orbital proptosis. Ear Nose Throat J 2007;86:23-4.

10. Na CY, Choi KS. A radiographic study of differential diagnosis between dentigerous cysts and unicystic ameloblastomas. Korean J Oral Maxillofac Radiol 1993;23:255-64.

11. Weber AL. Imaging of cysts and odontogenic tumors of the jaw. Definition and classification. Radiol Clin North Am 1993;31:10120.

12. Robinson L, Martinez MG. Unicystic ameloblastoma: a prognostically distinct entity. Cancer 1977;40:2278-85.

13. Som PM, Dillon WP, Curtin HD, Fullerton GD, Lidov M. Hypointense paranasal sinus foci: differential diagnosis with MR imaging and relation to CT findings. Radiology 1990;176:777-81.

14. Dagistan S, Cakur B, Goregen M. A dentigerous cyst containing an ectopic canine tooth below the floor of the maxillary sinus: a case report. J Oral Sci 2007;49:249-52.

15. Chuong R. Dentigerous cyst involving maxillary sinus: report of case. J Am Dent Assoc 1984;109:59-60.

16. Hyomoto M, Kawakami M, Inoue M, Kirita T. Clinical conditions for eruption of maxillary canines and mandibular premolars associated with dentigerous cysts. Am J Orthod Dentofacial Orthop 2003;124:515-20.

17. Ertas U, Yavuz MS. Interesting eruption of 4 teeth associated with a large dentigerous cyst in mandible by only marsupialization. J Oral Maxillofac Surg 2003;61:728-30. 